\title{
Reflets
}

Revue d'intervention sociale et communautaire

\section{Principes féministes et intégration théorie-pratique - Un parcours universitaire}

\section{Entrevue avec Cécile Coderre}

\section{Madeleine Dubois}

Volume 20, numéro 1, printemps 2014

La formation pratique : allier milieux de pratique et milieux

d'enseignement

URI : https://id.erudit.org/iderudit/1025793ar

DOI : https://doi.org/10.7202/1025793ar

Aller au sommaire du numéro

Éditeur(s)

Reflets, Revue d'intervention sociale et communautaire

ISSN

1203-4576 (imprimé)

1712-8498 (numérique)

Découvrir la revue

Citer ce document

Dubois, M. (2014). Principes féministes et intégration théorie-pratique - Un

parcours universitaire : entrevue avec Cécile Coderre. Reflets, 20(1), 18-40.

https://doi.org/10.7202/1025793ar 


\section{Principes féministes et intégration théorie-pratique - Un parcours universitaire}

\section{Entrevue avec Cécile Coderre}

Au cours de sa carrière à l'Université d'Ottawa, débutée en 1983, madame Cécile Coderre a occupé les postes de professeure de sociologie, de professeure et de directrice de l'École de service social et de vice-doyenne de la Faculté des sciences sociales. Peu importe la nature du poste qu'elle occupait, elle a toujours mis de l'avant les principes des approches féministes en reconnaissant, notamment, l'importance de tisser des liens entre les diverses formes de connaissances ainsi qu'entre les assises théoriques et les manifestations de savoirs pratiques. Son engagement au sein d'associations vouées au développement communautaire et au bienêtre des femmes, jumelé à la rigueur intellectuelle qui l'anime, vivifie son enseignement et nourrit sa réflexion sur les rapprochements qu'elle fait entre les diverses formes de savoirs.

L'entrevue a été réalisée par Madeleine Dubois.

M. Dubois : Nous nous intéressons dans cette entrevue à l'évolution de votre vision professionnelle en cohérence avec les valeurs féministes, ainsi qu'aux défis et enjeux rencontrés dans l'application de ces principes dans votre parcours universitaire. Commençons par votre formation universitaire de $1^{\text {er }}$ et de $2^{e}$ cycle en travail social. Qu'est-ce qui vous a incitée à vous inscrire en travail social et quel rôle les stages ont-ils eu dans votre formation initiale?

C. Coderre : J'ai fait mes études de baccalauréat en service social à l’Université Laval de 1974 à 1977. Mon intérêt pour la formation en service social a découlé de mon implication dans le mouvement étudiant au CÉGEP de Joliette. Au cours de mes études de CÉGEP, les mouvements étudiants étaient en ébullition et ils s'intéressaient, 
notamment, à la démocratie directe. J'avais plusieurs intérêts dans les domaines des sciences sociales et des sciences politiques. Et quoique j'aie été passionnée par mes cours de sociologie et de psychologie, je cherchais plutôt un programme universitaire où je pouvais faire du changement politique et social.Je participais à de nombreuses manifestations. C'était une époque où la majorité des étudiantes et des étudiants faisaient front commun et où le Québec était en évolution. Je voulais donc m'inscrire dans un programme d'études qui me permettrait de nourrir mes aspirations de changement, ce qui m'a amenée à fixer mon choix sur le programme de service social de l'Université Laval, qui offrait un domaine d'études sur l'action communautaire.

Les stages dans le domaine communautaire ont été remplis de défis associés aux particularités et aux raisons d'être du développement communautaire. Au cours des années 1970, il se faisait beaucoup de rénovation urbaine. On transférait des populations entières, un peu comme ce qui s'est fait dans la Basse-ville à Ottawa à la même époque. Je souhaitais mettre sur pied un projet bien défini avec une collègue stagiaire, mais nous avons rapidement constaté qu'il serait impossible de mettre à exécution toute la planification que nous avions faite si diligemment parce que les situations changeaient du jour au lendemain. J'ai fait un premier stage avec la centrale syndicale CSN, qui soutenait la création d'un village vacances-famille pour des travailleuses et des travailleurs de Québec. Notre projet initial a dû être transformé complètement pour s'adapter à la réalité du contexte. La même situation s'est répétée au cours de mon deuxième stage avec les comités de citoyens des HLM. Avec des collègues, nous avons alors décidé de créer une boite à outils pour l'animation communautaire. Il s'agissait d'outils techniques qui nous auraient été utiles pendant nos stages, présentés sous forme de fiches portant notamment sur l'incorporation des organismes, la rédaction d'ordres du jour et de procès-verbaux, la préparation de budgets et l'organisation d'une conférence de presse.

M. Dubois : Ces expériences vous ont permis d'apprendre en quoi consistait réellement l'intervention communautaire. 
C. Coderre : En effet,j'ai fait de nombreux apprentissages au cours de ces stages, non pas en réalisant concrètement ce que j'avais conceptualisé, mais en m'engageant véritablement. J'ai appris, notamment, à m'adapter au milieu, à être à l'écoute, à me montrer souple et ouverte aux changements et à tenir compte des points de vue de plusieurs personnes. J'ai ainsi fait des apprentissages extrêmement importants. Par ailleurs, comme l'université nous encourageait beaucoup à faire des stages communautaires avec d'autres étudiantes ou étudiants de l'école, j'ai donc travaillé en collaboration avec une collègue. La planification et la préparation aux stages s'effectuant en collaboration, nous avons pu développer nos aptitudes pour le travail d'équipe, raffiner nos capacités de coopération et de partage. Pour réussir, il fallait que notre projet soit développé en commun pour ensuite être adapté aux besoins de communautés particulières. Ce type de collaboration m'a beaucoup interpellée, m'amenant à effectuer des transformations personnelles, parce qu'en quelque sorte,j'ai émergé de l'étudiante très studieuse que j'étais. J'ai baigné dans la communauté et je me suis éveillée aux questions sociales, notamment à la pauvreté. C'était une réalité qui m'était jusqu'alors inconnue. Je reconnais sans contredit avoir beaucoup acquis des stages qui s'échelonnaient sur une année pendant ma formation universitaire.

Mes études de baccalauréat ont aussi concrétisé mon désir de continuer mes études au niveau supérieur, ne serait-ce que des inquiétudes que j'avais de commencer à travailler tout de suite après mon baccalauréat. Je reconnaissais la nécessité d'acquérir des habiletés et les stages m'avaient convaincue de l'importance de poursuivre ma réflexion. Il s'agit là d'ailleurs d'un sujet de préoccupation que nous partagent encore souvent nos étudiantes et nos étudiants aujourd'hui.

J'ai décidé de partir étudier en Europe parce qu'à l'époque, on offrait peu de programmes d'études supérieures en français au Canada, notamment dans les domaines de la sociologie ou du service social. Plutôt que d'étudier en anglais à l'Université de Toronto ou aux États-Unis, j'ai choisi d'aller en France, où 
j'ai poursuivi mes études de service social et de sociologie. En service social, mon programme s'intéressait davantage aux aspects familiaux. J'ai été introduite à des problématiques qui m'étaient jusqu'alors inconnues, notamment dans le contexte des stages que j'ai effectués dans des cliniques d'avortement à Lyon. Les problématiques liées à la question des femmes m'ont beaucoup interpellée. Par ailleurs, dans le cours de mes études de maitrise en sociologie, je me suis surtout intéressée à la rénovation urbaine et aux mouvements des citoyens, réunissant ainsi mes champs d'intérêt pour les mouvements sociaux avec une analyse plus théorique sur le logement social.

M. Dubois: Vos études de $2^{e}$ cycle en France vous ont donc permis de découvrir un intérêt pour les études des femmes, tout en élargissant vos connaissances sur les mouvements sociaux amorcées pendant vos études de baccalauréat en service social. Retenez-vous d'autres apprentissages de ces études qui ont marqué votre positionnement professionnel?

C. Coderre: J'ajouterais que lorsque j'ai fait mes études de baccalauréat en 1974-77, le mouvement des femmes était très peu présent dans les écoles de service social, même s'il l'était dans la communauté. J'aurais voulu faire un stage à la clinique de santé des femmes qui venait d'ouvrir à Québec, mais l'école a refusé parce qu'il n’y avait pas de superviseure attitrée, c'est-à-dire des travailleuses sociales professionnelles, à cette clinique. Malgré l'importance accordée aux stages, même dans le domaine du communautaire, les universités étaient réticentes à ce que le milieu communautaire soit en soi un élément formateur important de notre expérience d'apprentissage. Et l'ouverture à ce type d'apprentissage, je jugeais très important que nous en tenions compte lorsque je suis arrivée à l'École de service social de l'Université d'Ottawa plusieurs années plus tard. Mes réflexions suite à l'expérience de ne pas avoir pu faire mon stage dans les cliniques de santé des femmes ont profondément marqué ma façon de percevoir les liens entre l'université et son milieu. Ça m'a amenée à avoir une nouvelle ouverture par rapport à la place du communautaire dans la formation de nos étudiantes et de nos étudiants. 
M. Dubois : Dans quel domaine avez-vous poursuivi vos études de doctorat et de quelles façons celles-ci ont-elles nourri votre cheminement réflexif, particulièrement en ce qui a trait aux liens entre la théorie et la pratique?

C. Coderre : Quand j'ai entrepris des études menant à un doctorat, je travaillais encore sur les questions sociales, notamment la rente foncière, le capitalisme monopolitique d'État. Mais j'ai constaté que je n'étais pas suffisamment motivée par ces sujets pour y consacrer mes études de $3^{\text {e }}$ cycle. Je travaillais dans un champ purement théorique et très économiciste et même si ces approches avaient élargi mes horizons, elles ne me passionnaient pas. Même si je m'intéressais toujours aux mouvements sociaux, un sujet qui pouvait certainement amener une nouvelle réflexion sur le social, je ne voulais pas que mes études se limitent à des perspectives intellectuelles et théoriques. Après avoir fini une première partie de mon D.E.A., j'ai donc recentré mes recherches sur le mouvement des femmes qui, en France, avait commencé à prendre de l'ampleur. Je me suis intéressée au discours sur la maternité, à la critique féministe de la maternité et à son impact dans la société. Mes réflexions théoriques ont été beaucoup nourries par la parole des femmes auvrant dans les mouvements sociaux. Je travaillais sur les premiers écrits féministes, les revues féministes que les femmes ont créées dans ce mouvement.J'ai découvert une diversité de points de vue, allant des théories radicales aux théories socialistes et au marxisme. Tous les courants de pensée féministes, je les ai découverts dans les revues féministes, telles que Le torchon brûle et Le temps des femmes. Ces revues m'ont beaucoup aidée à comprendre comment analyser d'une perspective plus théorique le lien privé-public, la critique de la maternité, l'exploitation des femmes. J'ai commencé à intégrer une pensée théorique avec laquelle je me sentais plus à l'aise, se situant dans un courant qu'on nommait en Amérique, radical et en France, matérialiste. Ces courants ont concrétisé mon engagement envers la parole des femmes et raffiné les liens entre celui-ci et les réflexions théoriques développées dans mes cours universitaires.

M. Dubois : Vous avez donc approfondi votre intérêt pour la situation des femmes tout en renouvelant l'importance que vous accordiez aux connaissances 
théoriques, grâce à une nouvelle perspective. Pourrait-on dire qu'en reconnaissant l'importance d'alimenter les notions théoriques à partir de ce que les femmes vivaient, vous reconnaissiez la mutualité d'enrichissement possible entre la théorie et la pratique?

C. Coderre : Oui, d'autant plus que la critique féministe était alors en ébullition. Par exemple, toutes les questions de l'intersectionnalité discutées de nos jours étaient déjà présentes dans le mouvement des femmes. Les écrits issus du mouvement des femmes me nourrissent encore beaucoup, m'amènent à réaliser que je dois poursuivre mes apprentissages parce que les femmes ont tant à nous apprendre. Le mouvement des femmes a toujours eu cette capacité de jeter un regard critique sur ses pratiques, contribuant ainsi à leur transformation. La pensée a évolué, entrainant des transformations dans les pratiques issues de l'intervention féministe et en sens inverse, la critique que les femmes apportent sur les pratiques fait évoluer la réflexion du mouvement. Se dessine alors un mouvement constant de va-et-vient qui sert de nourriture intellectuelle autant pour les pratiques que pour les réflexions théoriques.

M. Dubois: Après quelques années comme professeure de sociologie à l'Université d'Ottawa, vous avez orienté votre carrière d'enseignement vers le service social, d'abord en contribuant activement à la création de l'École de service social de l'Université d'Ottawa au début des années 1990. Il s'agissait de mettre sur pied un programme francophone de deuxième cycle en service social donné uniquement en français, ce qui était assez unique. L'Université Laurentienne à Sudbury offrait depuis quelques années un programme en français, mais il n'en demeure pas moins qu'en Ontario, offrir un programme de $2^{e}$ cycle donné uniquement en français était vare à cette époque. Quel rôle avez-vous voulu jouer dans la création de cette nouvelle formation, et comment votre travail précédent a-t-il influencé votre façon d'envisager ce programme, qui était en quelque sorte historique pour le milieu franco-ontarien qui revendiquait depuis longtemps la nécessité de formation de professionnelles et de professionnels travaillant en français?

C. Coderre : Lorsque j'ai été embauchée en 1983 à l'Université d'Ottawa, le doyen s'est montré très intéressé au fait que j'avais des formations à la fois en sociologie et en service social. Depuis la publication du 
Rapport Dubois de 1976 sur la situation en matière de santé en Ontario français (Gilbert, et collab., 2005), la communauté francoontarienne faisait des représentations à l'Université d'Ottawa, l'incitant fortement à développer des programmes en santé et en service social en français. En 1985, à la suite de la publication du Rapport du groupe de travail sur les services universitaires en français offerts par l'université, connu sous le nom de "Rapport Carrier ", le projet de développement d'une formation en service social est lancé. Il importe aussi de souligner la conjoncture favorable créée par l'impact crucial de la Loi ontarienne de 1986 sur les services en français. Or, très peu de professeurs avaient la formation nécessaire pour enseigner le service social et, au sein de l'université, on retrouvait peu de ressources pour amorcer la création d'une école de service social. Lorsqu'on m'a approchée pour participer à la création de ce programme, j'étais très engagée déjà en études des femmes puisque je m'étais grandement investie dans ce programme, commencé l'année précédant mon embauche. Par ailleurs, comme j'avais préparé en 1984 et en 1985 les rapports d'équité sur la situation des femmes professeures à l'Université d'Ottawa et ceux relativement aux employées et employés administratifs et aux étudiantes et étudiants, j'avais été mandatée avec trois autres femmes de l'Université de créer les premiers programmes d'équité à l'Université d'Ottawa. Parallèlement à mes tâches universitaires, j'étais aussi très engagée dans le mouvement féministe au Québec et dans le mouvement des femmes contre la pornographie. Par ailleurs, le Réseau national d'action et d'éducation des femmes m'avait approchée pour effectuer avec Linda Cardinal une recherche-action sur les besoins des femmes en milieu minoritaire en matière d'éducation. Nous avions alors visité de nombreuses communautés francophones canadiennes en contextes minoritaires, de Terre-Neuve jusqu'à Victoria. Les femmes de toutes ces communautés nous ont amenées à constater l'importance cruciale des enjeux liés à l'éducation postsecondaire pour les communautés minoritaires de même que de la contribution des femmes francophones à leur communauté.

M. Dubois : Dès les débuts de votre carrière, vous étiez donc engagée dans plusieurs activités et projets de recherches présentant un grand intérêt autant pour 
le travail social que pour les études des femmes, et qui posaient en quelque sorte les jalons de votre contribution dans le développement de l'Ecole de service social.

C. Coderre: De façon générale, les recherches que j'effectuais avec les mouvements de femmes, par exemple celle contre la pornographie avec la Fédération des femmes du Québec, ont toujours adopté des modèles de recherche-action. J'ai privilégié ce modèle de recherche tout au long de ma carrière.

Lors de la création de l'École de service social, j'ai constaté que le programme de service social se prêtait très bien à la rechercheintervention, et qu'un tel programme ralliait tous mes sujets de prédilection, notamment ceux développés dans le cadre des projets sur les minorités francophones. Dès la conception de l'École de service social, la vision pour celle-ci, qui avait été élaborée par un comité sous la direction de Roland Lecomte, accordait une place prépondérante au développement de projets de rechercheintervention avec la communauté francophone de la région. Nous avions à cœur que cette école soit celle de la communauté et que les différentes communautés francophones minoritaires, plus particulièrement en Ontario, aient accès à des études postsecondaires et à des études supérieures, afin de transformer leur place dans la communauté. C'était une question de droit.

M. Dubois : La communauté franco-ontarienne a bien soutenu ce projet, $y$ a participé et contribué de diverses façons. Ses représentantes et représentants, notamment des milieux communautaires et de la santé, ont contribué à nourrir la réflexion sur la conceptualisation d'une nouvelle école, ce qui était plutôt rare dans les milieux universitaires.

C. Coderre : Si la communauté n'avait pas été là, l'université n'aurait probablement pas mis sur pied ce programme. L'université était très sensible aux pressions de la communauté franco-ontarienne. De plus, au sein des membres de son administration, certaines personnes, dont monsieur Denis Carrier et madame Caroline Andrew, alors vice-doyenne à la recherche, ont joué un rôle très important pour soutenir à l'intérieur de l'institution la création d'un programme s'offrant uniquement en français. Lors 
de la mise sur pied de l'école, lorsque nous avons effectué une étude pour identifier des intervenantes et intervenants de la communauté aptes à superviser les stages, nous avons constaté la grande pénurie de personnel francophone formé en service social. Influencée par mon expérience personnelle d'étudiante de baccalauréat en service social, j'étais convaincue de l'importance de collaborer avec des communautés qui nous ouvraient leurs portes, notamment pour trouver des solutions de rechange à la pénurie de superviseurs de stage. Tout comme elle l'a fait pour la création de l'école, la communauté franco-ontarienne nous a appuyés dans les mouvements de pression pour combler les lacunes dans les ressources. Soulignons qu'il ne s'agissait pas seulement de certains représentants de la communauté, mais bien de la communauté. Un tel soutien est exceptionnel dans les milieux universitaires.

M. Dubois : Revenons à la situation que vous avez évoquée au sujet de la nécessité d'avoir des gens formés en service social pour superviser des stagiaires en service social.Vu les lacunes de personnel formé au sein de la communauté francophone et afin ne pas perdre de vue la vision de l'École, il fallait tenir compte d'autres facteurs importants : l'expérience des personnes francophones qui travaillaient déjà dans la communauté, la variété et la richesse des connaissances et des savoirs qu'elles avaient développés. Il n'était pas question de se laisser définir uniquement en fonction des diplômes exigés pour la supervision par les associations professionnelles.

C. Coderre: C'était l'ouverture qu'avait alors l'École et qu'on a continué d'avoir. Aujourd'hui, nous avons réussi à former un bassin important de personnes francophones qui ont maintenant un diplôme de maitrise en service social. Nous avons en quelque sorte enrichi la communauté.

M. Dubois : En effet, ce n'est pas rare d'aller dans des organismes francophones et d'y rencontrer des travailleuses sociales et des travailleurs sociaux diplômés de l'Université d'Ottawa.

C. Coderre: J'ajouterais même que ces diplômées et diplômés travaillent désormais dans diverses régions de l'Ontario, dans le Sud-ouest, par exemple. Il y a des communautés ontariennes qui ne peuvent 
pas être désignées en vertu de la Loi sur les services en français parce qu'elles ne comptent pas le nombre suffisant de francophones exigé pour une telle désignation, mais qui peuvent néanmoins offrir quelques services, notamment en matière de violence faite aux femmes, grâce à l'augmentation du nombre de professionnelles et de professionnels formés en français. Il s'agit d'une avancée malgré les nombreuses limites toujours présentes.

M. Dubois : Les questions et enjeux liés à l'intégration de la théorie et de la pratique au sein d'études universitaires, plus particulièrement de deuxième cycle, ont fait l'objet de nombreuses réflexions et discussions depuis quelques années. Lorsque vous avez commencé à enseigner en service social en 1993, la réflexion sur ces questions était moins articulée, mais elle soulevait néanmoins des débats et des tensions, dont certains sont d'ailleurs toujours présents. Les étudiantes et étudiants ayant suivi vos cours ont souvent témoigné d'activités provenant des pratiques qui y ont été intégrées et qui les ont beaucoup inspirés. Quelles approches théoriques et quelles activités issues des pratiques vous ont inspirée pour faciliter les liens entre la théorie et la pratique dans le contenu de vos cours?

C. Coderre : À l'époque où j'entreprenais ma carrière d'enseignement universitaire dans les années 1980, les groupes de conscientisation occupaient une place importante au sein des mouvements de femmes. Ces groupes travaillaient toujours avec des outils de communication dont on se sert encore aujourd'hui dans les Centres d'aide et de lutte contre les agressions à caractère sexuel (CALACS) et dans certaines maisons d'hébergement. Nommons à titre d'exemple le "comment ça va? ", des outils de communication relativement au ressentiment, à la communication des malaises. Dans le cours universitaire d'intervention féministe, nous appliquions ces pratiques afin de conscientiser les étudiantes qui travailleraient plus tard dans les groupes de femmes ou dans les services pour les femmes à être en mesure d'appuyer leurs interventions sur des réflexions fondées dans les réalités vécues par les femmes. Nous avions aussi adopté le mode de fonctionnement d'une collective, en tentant de remettre en cause le statut inégal des relations professeure-étudiante. Nous voulions également préparer les étudiantes en vue de répondre aux besoins de main-d'œuvre 
et de formation dans les maisons d'hébergement et les CALACS en élaboration. Comme plusieurs de ces ressources offraient du soutien aux femmes ayant subi diverses formes de violence, je me servais aussi, dans mes cours, des guides rédigés par Ginette Larouche $(1985 ; 1987)$ qui avait travaillé avec la collective Par et Pour Elle à Montréal, la première collective féministe adressant les questions de violence faite aux femmes. Créés originalement pour les groupes de soutien aux femmes qui vivaient la violence, ces textes ont été largement utilisés dans les cours d'intervention féministe.

Par la suite, après plusieurs années pendant lesquelles j'ai été absente de l'enseignement vu les tâches administratives que j'ai assumées à l'université, je me suis inspirée à nouveau de ces mêmes liens avec les pratiques lorsque je suis revenue dans l'enseignement. Je suis allée dans les différentes ressources francophones pour les femmes d'Ottawa et de l'Est de l'Ontario pour me renseigner sur leurs outils d'intervention; mon but était que les étudiantes et les étudiants inscrits aux cours d'intervention féministe, de problématique de la violence et de problématique familleenfance soient sensibilisés à l'utilisation des outils d'intervention. Je demeure toujours intéressée à l'intégration de ces outils dans un plan de cours universitaire, afin d'amener les étudiantes et les étudiants non seulement à les connaître, mais à y réfléchir en les confrontant à des concepts théoriques et, à la longue, peut-être à les raffiner. Dans mes cours, j'invite aussi les étudiantes et les étudiants à lire de nombreux témoignages de femmes. Il y a par exemple le témoignage d'une femme dont les enfants ont été assassinés par son conjoint. Cette femme relate ce qui s'est passé et comment les services sociaux n'ont pas su répondre à ses besoins. Et plus récemment,j'utilise les témoignages entourant l'affaire Shafia pour questionner l'intervention auprès des jeunes filles immigrées et issues de communautés où le mariage forcé est coutumier. Nous analysons leurs propos afin de déceler les lacunes dans les services et proposer des pistes pour y remédier. Ces témoignages ont un impact profond sur les étudiantes et les étudiants. Nous faisons par la suite des liens avec des textes théoriques, par exemple celui de Nicole Claude Mathieu intitulé Quand céder n'est pas 
consentir, publié en 1985, qui demeure un texte très puissant sur les contraintes vécues par les femmes et sur la violence comme forme de contrôle social. Après avoir été confrontés et touchés par les témoignages des femmes, les étudiantes et les étudiants abordent la lecture et l'analyse des textes théoriques avec une perspective plus éclairée, et ce, malgré la complexité parfois grande de certains de ceux-ci. Les commentaires des étudiantes et des étudiants réitèrent toujours pour moi l'importance de faire des liens dans mon enseignement entre les textes théoriques, les pratiques d'intervention connues et celles qui sont en émergence, soit dans les milieux ou dans le bagage d'outils personnels que chaque intervenante ou intervenant est appelé à développer dans sa pratique.

M. Dubois : Cette confrontation de la théorie à la pratique éveille les étudiantes et les étudiants à des réalités qui resteraient occultées s'ils restaient uniquement centrés dans la théorie. Les témoignages personnels peuvent remuer les interlocuteurs et susciter des apprentissages différents de ceux émanant de la lecture de textes théoriques ou d'études empiriques. Il semble en quelque sorte que les apprentissages axés sur l'intégration entre la théorie et la pratique sont plus marquants.

C. Coderre: C'est d'ailleurs la méthode utilisée par Pierre Bourdieu dans les études qu'il a effectuées et dont il rend compte dans La misère $d u$ monde. Il est allé rencontrer des gens et leur a dit : «Racontezmoi votre vie. "C'est à partir de leurs propos et du processus de réflexivité amorcé sur ces échanges que l'auteur a construit des concepts théoriques. Il s'agit d'un exemple percutant de lien théorique articulé à partir de l'expérience ou du vécu de personnes. Ces liens dans le développement de savoirs que je tente de transmettre à mes étudiantes et à mes étudiants, je les appuie sur la parole de femmes, car je crois que ce type d'enseignement facilite l'émergence de nouvelles dimensions, façonnées dans des rapports d'intersectionnalité tenant compte des complexités de la vie.

M. Dubois : En discutant de vos responsabilités professionnelles universitaires, vous avez mentionné l'importance que vous accordez à votre implication communautaire. Dans quels domaines vous êtes-vous engagée et comment 
cet engagement a-t-il nourri votre enseignement et votre positionnement comme professeure?

C. Coderre : Dans ma carrière professionnelle,j'ai beaucoup tenté de contribuer à la valorisation de la recherche-intervention, en m'engageant avec différents groupes francophones vivant en contexte minoritaire, notamment avec la communauté franco-ontarienne. J'ai aussi souvent travaillé en étroite collaboration avec la Fédération nationale des femmes canadiennes-françaises, maintenant appelée Alliance des femmes de la francophonie canadienne. Avec l'Alliance, je me suis engagée dans un projet de recherche-action entre 2001 et 2003 intitulé Des droits sociaux pour les femmes francophones en contexte minoritaire, une lutte pour éradiquer la pauvreté. Nous avons rencontré les femmes des diverses communautés afin de développer avec elles un argumentaire politique pour faire valoir ce que nous avons nommé un "revenu de citoyennes". Les femmes vivant dans la pauvreté récusaient les connotations péjoratives associées au panier de consommation ou au revenu minimum et revendiquaient plutôt un revenu de citoyenneté. Notre travail avec les participantes nous a amenées, notamment, à démystifier les politiques sociales, à identifier leurs répercussions sur les femmes, à discuter des liens entre la sécurité alimentaire et la santé et à développer des outils pour revendiquer auprès du gouvernement canadien qui a signé des ententes internationales de lutte à la pauvreté. Le but à long terme était de soutenir et d'outiller les femmes francophones des différentes régions du Canada pour qu'elles soient plus aptes à reconnaître les causes structurelles de la pauvreté et qu'elles deviennent porteuses du dossier de lutte à la pauvreté dans la communauté francophone. Comme notre travail s'effectuait avec des personnes d'une variété de milieux et de contextes géographiques, s'étendant de TerreNeuve aux Territoires du Nord-Ouest, il fallait se sensibiliser aux circonstances et aux défis particuliers de chacun des groupes, créer avec les femmes des ateliers basés sur leur réalité provinciale ou territoriale tout en adaptant des connaissances plus larges eu égard, par exemple, à la loi ou au processus électoral. Le plan politique résultant de ce travail a été livré au Congrès mondial acadien tenu à Halifax. Ce projet a permis un changement social au sein 
d'une communauté qui s'exprimait peu sur la place publique. Les femmes pauvres ne sont pas très écoutées dans notre société, leur voix n'est pas souvent entendue et au sein de ce projet, elles ont développé un cahier de revendications. Dans cette même période, j'ai préparé avec la Fédération nationale des femmes canadiennesfrançaises le premier rapport sur les services en français en violence dans les communautés minoritaires au Canada intitulé Des services en français pour les femmes vivant des situations de violence, un droit fondamental. C'est important pour moi, en tant que professeure universitaire, de faire alliance avec des groupes marginalisés et j'opte pour des groupes de femmes. J'ai rencontré des femmes extraordinaires dans ces recherches, des femmes engagées et inspirantes.

Le projet sur la pauvreté et les politiques sociales que j'ai réalisé avec Madeleine Roy a aussi donné l'occasion de créer différents outils de conscientisation, dont le Budget déséquilibrant et un jeu de Serpents et d'échelles que j'utilise dans mes cours. Ceux-ci sont en voie d'être actualisés par Joscelyne Levesque avec des assistantes de recherche et ils seront disponibles en ligne pour des fins pédagogiques.

M. Dubois : Poursuivez-vous toujours votre travail communautaire avec des groupes de femmes?

C. Coderre : Je suis actuellement engagée dans un projet avec la Coalition de Prescott-Russell contre la violence faite aux femmes. L'année dernière, les intervenantes d'Action ontarienne contre la violence faite aux femmes m'ont mise en contact avec la Coalition de Prescott-Russell qui, à la suite de questions soulevées par un journaliste au sujet du nombre de femmes vivant de la violence et de l'utilité des services leur étant offert, a décidé de recueillir des données et de rédiger un rapport de documentation. Depuis 2002 , très peu près de recherches ont porté sur la violence faite aux femmes en milieu rural au Canada, ce qui explique les lacunes dans la connaissance de cette problématique. Les femmes souhaitaient de plus approfondir le travail de leur table de concertation, ce qui a mené au développement d'un autre projet auquel s'est intégrée une étudiante de Prescott-Russell rédigeant 
son mémoire de maitrise sur la réalité des jeunes femmes en milieux ruraux. La participation d'étudiantes et d'étudiants dans nos projets de recherche-action offre une excellente opportunité d'approfondissement des liens entre la théorie et la pratique. Nous avons choisi d'appuyer notre projet sur une méthodologie qui pourrait être utilisée dans différentes régions de l'Ontario, afin de recueillir des données plus spécifiques. Pour ce faire, nous nous sommes inspirées dans notre démarche méthodologique, d'une recherche communautaire réalisée par la Coalition d'Ottawa contre la violence faite aux femmes, intitulée À l'abri des regards, volume II Prévalence de la violence faite aux femmes à Ottawa, à laquelle nous avons donné une couleur spéciale. En nous basant sur le contenu de mes cours universitaires, nous avons identifié les connaissances jugées utiles pour appuyer les demandes de subvention faites par les groupes communautaires, ce qui nous a amenées à mettre à jour les données statistiques disponibles sur la violence faite aux femmes vivant en milieu rural en Ontario et à présenter de manière synthétique l'état actuel des connaissances sur les violences faites aux femmes. Nous souhaitions en même temps offrir des outils pour répondre aux très grands besoins de formation des femmes en milieu rural, qui y ont rarement accès. Je considère donc qu'il est essentiel de partager les connaissances que j'acquiers comme professeure et comme chercheuse, non seulement avec les étudiantes et les étudiants, mais aussi avec la communauté. C'est dans cette optique que je choisis un engagement qui soutient la cause qui est celle des femmes.

M. Dubois : Il n'est pas nécessairement aisé pour un professeur ou un chercheur universitaire de se faire accepter par les milieux communautaires. On peut être perçu comme un intrus, voire comme persona non grata. Quels attitudes et moyens mettez-vous de l'avant pour vous faire accepter, vous intégrer dans des milieux qui ne vous connaissent pas?

C. Coderre : Effectivement, nous sommes souvent perçus comme œuvrant dans une tour d'ivoire. Cela peut soulever au sein des communautés l'inquiétude qu'on imposera nos connaissances et qu'on agira à la place des gens du milieu. Je m'assure donc de me montrer très ouverte, d'obtenir beaucoup de rétroaction des personnes avec 
qui je travaille en leur proposant des plans préliminaires. On s'entend dès le début sur des paramètres très clairs, par exemple, la distribution des tâches et qui signera le rapport final. Les personnes avec qui je travaille sont mes alliées et non des sujets de recherche. La recherche féministe m'a appris à quel point il est essentiel de ne pas avoir de jugement, de savoir écouter les femmes qui sont toujours les expertes de leur vie. Il leur appartient donc de décider des conclusions, des recommandations de nos rapports. Ce sont elles qui décident que certaines données seront publiées ou pas, qui choisissent les enjeux politiques qu'elles jugent importants. Le respect pour leurs décisions doit être clairement articulé au début de chaque projet de recherche et constamment renforcé dans notre positionnement de travail.

M. Dubois : Vous avez souligné l'importance des valeurs féministes, du service à la communauté, en lien avec le savoir-être essentiel à l'exercice de la profession de travail social et discuté de certains défis et enjeux rencontrés dans votre engagement communautaire à titre de professeure. Lorsque vous avez occupé le rôle de directrice de l'École de service social, de 1998 à 2003, comment avez-vous mis de l'avant ces valeurs et principes?

C. Coderre : Mon rôle comme directrice consistait notamment à veiller à l'actualisation de la vision de l'École. Celle-ci a d'abord été créée dans le but de former des travailleuses sociales et des travailleurs sociaux afin de combler les lacunes dans les services s'adressant à la communauté francophone de l'Ontario. Nous avons donc développé de nombreux outils susceptibles de soutenir le travail de nos diplômées et diplômés dans la communauté. Nous avions, par exemple, mis sur pied à l'intérieur de certains cours un volet portant sur la dévictimisation, afin d'offrir aux étudiantes et aux étudiants un espace de liberté et de prise de parole facilitant une certaine conscientisation. Nous organisions aussi des conférences avec la collaboration et la participation de la communauté et les intervenantes et intervenants du milieu étaient régulièrement invités à participer aux cours, afin de sensibiliser les étudiantes et les étudiants aux grands enjeux de la pratique. Nous avons de plus développé un projet de collaboration avec la Cité collégiale afin d'ouvrir à ses diplômées et diplômés une voie 
vers des études de maîtrise en service social en français, d'où la création du programme en Intervention sociale. Comme il m'importait de garder vivante cette vision de l'importance des liens avec la communauté, je pouvais en tant que directrice soutenir des projets pour faciliter son actualisation. Ma tâche a été facilitée du fait qu'à l'époque l'université valorisait davantage qu'aujourd'hui les trajectoires universitaires se démarquant de la norme établie. La création de la revue Reflets, fondée dans une vision de collaboration entre universitaires et la communauté, constitue un bon exemple de la place accordée par l'université à la reconnaissance de projets novateurs. Je dirais qu'à certains égards, être directrice de l'école dans le temps où j'ai occupé ce poste était plus facile qu'aujourd'hui parce que la volonté qu'avait l'université de créer des liens avec sa communauté allait dans le même sens que la mission de l'École. Mais en se dirigeant vers une vision institutionnelle valorisant davantage la recherche subventionnée, l’Université s'est détachée peu à peu des projets de recherche-intervention. Ça devient donc plus difficile de soutenir à la fois une carrière professorale axée sur les buts de l'université et de nourrir une école avec une mission d'engagement avec la communauté.

M. Dubois : Malgré tout le travail consacré à la création d'un programme universitaire avec une mission prônant la valorisation du travail et des projets en partenariat avec la communauté et en dépit de certaines avancées en la matière, il n'en reste pas moins que les valeurs qui sous-tendent ce type de programme doivent constamment être réitérées et le travail pour y parvenir toujours renouvelé.

C. Coderre : Je dirais en effet que les conditions sont plus difficiles dans le système universitaire aujourd'hui. Même si la mission de notre école se veut différente de celle d'autres départements, les professeures et les professeurs ont à répondre aux mêmes conditions que celles exigées de l'ensemble de leurs collègues. Mais la notion même de partenariat de recherche en collaboration avec les communautés est moins valorisée à l'université. Les attentes relatives à la recherche sont axées sur de l'autofinancement alors que lorsqu'on travaille dans des organismes communautaires le financement est 
rare et limité. La plupart des groupes communautaires, les groupes de femmes avec qui je travaille, entre autres, sont incapables de financer une recherche dont ils ont pourtant besoin pour continuer d'exister. Ces groupes sont pris dans un cercle vicieux : l'obtention de financement doit s'appuyer sur des données de recherche, mais ils n'ont pas les ressources pour effectuer ces recherches, ni les moyens d'en financer le coût. À mon avis, nous avons une responsabilité en tant que chercheurs d'aider ces organismes qui sont excessivement démunis économiquement à développer les données dont ils ont besoin pour poursuivre leur travail.

Il est aussi important de souligner que les étudiants et les étudiants sont aussi perdants lorsque leur université cesse d'appuyer la recherche-intervention avec les communautés, parce que participer à de tels projets et en entendre parler dans ses cours mettent en lumière certains liens entre la théorie qu'ils apprennent et les réalités de leur vie. Par exemple, lorsque je donne des exemples de Prescott-Russell dans mes cours, les étudiantes et les étudiants qui connaissent cette région font des liens avec ces données qui prennent alors une signification plus vivante, avec des points de repère qui leur sont familiers. Ils se sentent mieux outillés et sont plus aptes à faire des changements au sein de leur communauté. En somme, ces approches prodiguent un sens important à l'enseignement, dynamisent les classes et enrichissent le contenu des cours.

M. Dubois : Au cours de votre carrière universitaire, vous avez également occupé le poste de vice-doyenne de la Faculté des sciences sociales à l'Université d'Ottawa. De quelles façons vos valeurs féministes, les valeurs prônées par le service social, et votre vision des liens entre théorie et pratique vous ont-elles influencée dans ce rôle qui en était essentiellement un d'administration?

C. Coderre : En acceptant le poste de vice-doyenne des études de premier cycle, je souhaitais contribuer à rendre plus significative l'expérience étudiante. Je voulais que les décisions relevant de l'administration soient pensées en fonction des étudiantes et des étudiants et non basées uniquement sur leur portée administrative. J'étais animée par ma propre expérience d'étudiante universitaire où 
j'avais apprécié mes stages et les apprentissages sur le terrain. J'ai pu ainsi appliquer les connaissances issues de mes cours et les remettre en question en les confrontant aux réalités de mes milieux de stage, pour ensuite constater les améliorations nécessaires à apporter dans les milieux communautaires. Ces expériences m'ont permis de raffiner et d'exprimer mon propre jugement, d'appliquer des connaissances, mais aussi de les évaluer. En tant que vice-doyenne, j'ai donc choisi de travailler à créer de nouvelles possibilités d'apprentissage et à faire valoir l'importance d'enrichir l'expérience étudiante en insérant dans le cadre des programmes d'étude une variété de possibilités de travailler sur le terrain.J'ai aussi consacré beaucoup d'énergie au développement des stages internationaux, en bâtissant des formations prédépart, des programmes postdépart et des mécanismes d'évaluation, ainsi qu'en revendiquant la création de bourses spéciales pour les étudiantes et étudiants, pour qui ce type de stage occasionnait des frais d'études supplémentaires. En m'inspirant de l'expérience de l'École de service social, j'ai travaillé à la création de cours de recherche-terrain offerts dans divers pays où se déroulaient les stages, permettant ainsi aux stagiaires d'obtenir des équivalences de cours. Nous avons aussi créé un cours de projet de recherche s'adressant aux étudiantes et aux étudiants de quatrième année voulant s'intégrer dans les projets de recherche de professeures et des professeurs, et ce, dans le but de bâtir des ponts entre le baccalauréat et la maitrise et de faciliter le travail d'intégration entre les apprentissages théoriques et le travail sur le terrain. Ce cours était offert dans tous les programmes de la Faculté. Enfin, nous avons développé de nouveaux programmes d'études interdisciplinaires, notamment Études des conflits et droits humains, dont le contenu est nourri par les cours terrains et les recherches des professeures et professeurs.

En somme, notre travail a beaucoup cherché à enrichir les expériences d'apprentissage, à développer des occasions favorables au tissage de liens entre la théorie et la pratique. Dans cette optique, nous avons de plus beaucoup valorisé et soutenu l'engagement communautaire, un programme qui existait depuis quelques années à l'Université d'Ottawa. 
M. Dubois : Pourriez-vous expliquer en quoi consiste l'engagement communautaire faisant partie d'un cours universitaire?

C. Coderre: Il s'agit d'intégrer à un cours des heures de travail communautaire qui sont évaluées comme l'une de ses exigences. Les étudiantes et les étudiants s'engagent donc à effectuer un nombre prescrit d'heures de bénévolat dans un organisme communautaire à la suite desquelles ils rédigent un travail de réflexion portant sur leur expérience et qui est noté pour la réussite du cours. Il s'agit essentiellement de créer des ponts entre l'université et la communauté et de valoriser les autres sources pertinentes d'apprentissage comme compléments à celles résultant d'un enseignement formel.

M. Dubois : À cette étape-ci de votre cheminement professionnel et universitaire, quelle analyse faites-vous de l'évolution de la formation universitaire en travail social, eu égard notamment aux aspirations des étudiantes et des étudiants qui s'y inscrivent et à la vision de la pratique qui se reflète dans les enseignements?

C. Coderre: Je dirais d'emblée que mon appréciation se fonde sur un constat de confiance et d'optimisme, mais aussi de préoccupation. Dans les cours que j'enseigne, je suis ravie de constater à quel point les étudiantes et les étudiants sont passionnés de justice sociale et motivés à mettre fin aux oppressions et à la discrimination. Dans mon cours d'intervention féministe, j'exige des étudiantes et les étudiants qu'ils s'engagent dans un exercice de conscientisation comme il s'en fait au sein des groupes du mouvement des femmes. Ils doivent donc présenter un atelier de quinze minutes sur un sujet de leur choix, s'adressant à des jeunes de $10^{\mathrm{e}}$ et $11^{\mathrm{e}}$ année. Je suis impressionnée par leurs connaissances, la capacité qu'ils ont de s'adapter à la réalité des jeunes, de choisir des moyens pertinents pour les toucher. Les problématiques abordées sont variées, allant de l'homophobie dans les écoles secondaires à la pauvreté des étudiantes universitaires. Dans le cadre de ce travail, la rencontre et les discussions avec des personnes ayant vécu la problématique dont ils traitent les amènent se sentir solidaires d'elles. Ces exercices sont toujours très enrichissants, je suis touchée par les compétences et les passions exprimées par les étudiantes et les étudiants. 
Quant à mes préoccupations au sujet de l'état actuel de la formation en travail social, elles sont liées au discours corporatiste qui se manifeste aujourd'hui dans les écoles de service social. Les ordres professionnels, pour satisfaire à certaines instances gouvernementales, voudraient imposer désormais des examens à caractère normatif aux diplômées et diplômés, ce qui définirait des champs de pratique très étroits en service social. Dans certaines provinces, ces exigences corporatistes sont en voie de réalisation, obligeant les écoles de service social soucieuses de maintenir leur agrément à offrir des formations s'y conformant. La profession y perdrait à mon avis, parce qu'on risquerait fort de perdre la passion pour la justice sociale, qui demeure selon moi la plus grande valeur du service social. En effet, il pourrait s'avérer difficile d'intégrer des exercices tels que ceux évoqués touchant la conscientisation, par exemple, dans un contenu de cours axé sur une formation et des connaissances utilitaires et techniques. Les champs de pratique du travail social sont complexes, axés davantage sur l'importance de l'écoute des personnes et des besoins exprimés par les communautés, ce qui risque de se perdre dans des pratiques professionnelles déterminées par des normes corporatistes qui définiraient des champs de compétence en fonction de diagnostics.

Par ailleurs, je me préoccupe aussi de l'avenir de la recherche-action et de la recherche communautaire au sein de notre université. Si de telles recherches ne sont pas davantage financées ou reconnues par l'institution, les professeures et les professeurs pourraient se voir obligés d'opter pour des orientations de recherche plus traditionnelles, moins engagées, donc moins engageantes pour les étudiantes et les étudiants, et aussi moins redevables aux communautés. Ce serait une grande perte, notamment pour la communauté franco-ontarienne, qui a été un partenaire et un allié à la source de la création de notre école de service social, et qui, en dépit du chemin parcouru, est encore confrontée à nombreux obstacles dans sa lutte pour l'accès équitable à des soins de santé et de services sociaux. 
M. Dubois : Compte tenu de cette analyse riche d'expériences dans le domaine, comment entrevoyez-vous les perspectives d'avenir pour le service social et quels souhaits formulez-vous pour l'enseignement du service social?

C. Coderre: De nombreuses pistes s'offrent à nous pour le renouvellement d'approches d'enseignement, de formes d'apprentissage. Je pense, par exemple à l'approche basée sur les études de cas. On veut susciter la réflexion et la pensée critique chez les étudiantes et les étudiants, mais les cours axés majoritairement sur le transfert de connaissances théoriques et techniques n'offrent pas le modèle idéal pour y parvenir. Un modèle d'apprentissage par études de cas dans nos cours ouvrirait la voie à une formation beaucoup plus riche.

M. Dubois : Et voulez-vous exprimer des souhaits pour la profession du service social?

C. Coderre : Le service social devrait rester fidèle à son histoire, à ses valeurs, qui sont celles de la justice sociale, de l'analyse des formes de marginalisation et d'exclusion sociale. Il ne faut pas non plus perdre de vue l'importance des questions liées aux situations que vivent les femmes, ou que la question du sexisme soit délaissée parce que ces problématiques ont toujours leur importance dans notre société. L'étude des formes d'oppression vécues par les femmes, les femmes immigrantes, les femmes marginalisées a été au cœur de la création du service social. Il importe donc de garder le cap sur nos racines et ne pas oublier que malgré les progrès obtenus grâce aux luttes des femmes, les rapports sociaux de sexe demeurent une problématique importante dans nos sociétés.

M. Dubois: Je vous remercie chaleureusement, Madame Coderre, d'avoir partagé les réflexions se dégageant de votre cheminement professionnel riche d'expériences et d'apprentissages.

\section{Bibliographie}

BOURDIEU, Pierre, dir. (1993). La misère du Monde, Paris, Éditions du Seuil, 1460 p.

GILBERT, Anne, et collab. (2005). "Les discours sur la santé des organismes franco-ontariens : du rapport Dubois à la cause Montfort " Reflets : revue d'intervention sociale et communautaire, Vol. 11, No 1, p. 20-48. http://www.erudit.org/revue/ref/2005/v11/n1/013058ar.pdf 
LAROUCHE, Ginette (1985). Guide d'intervention auprès des femmes violentées, Montréal, Corporation des travailleurs sociaux du Québec, 93 p.

LAROUCHE, Ginette (1987). Agir contre la violence Une option féministe à l'intervention auprès des femmes battues, Montréal, La pleine lune, $549 \mathrm{p}$.

MATHIEU, Nicole-Claude ([1985] 1991). "Quand céder n'est pas consentir. Des déterminants matériels et psychiques de la conscience dominée des femmes, et de quelques-unes de leurs interprétations en ethnologie ", dans L'Anatomie politique, Paris, Côté-Femmes, p. 131-225. 